\title{
INTERNET AS A NEW TECHNOLOGICAL POSITION IN EDUCATION
}

\author{
Preecha Phongpheng \\ Suan Sunandha Rajabhat University, Bangkok, Thailand
}

Internet has become an everyday tool of people and organizations and, at the same time, indispensable to the proper functioning of business. With the growing increase in network infrastructures and mass popularization of the high-speed network, a breakthrough has emerged related to the use of the Internet, making it a global platform to allow intelligent machines and objects to communicate autonomously. This possibility allows content and services to be around people, always available, facilitating communication and opening the way for new applications; enabling new forms of work, interaction and entertainment; developing a new standard of living and work. This new standard is made possible by advances in Information and Communication Technologies (ICT) to a new concept defined as Internet of Things - IoT. This paper presents and discusses the main features that characterize the Internet of Things, its origin, theoretical visions and fields of application, exploring the possibilities of fomenting a discussion about the application of IoT.

Keywords: Internet of Things; technologic innovation; IOT; information and communication technologies

\section{Introduction}

Millions of people worldwide are using the Internet today on a daily basis to communicate, surf the Web, searching for various useful or fun content and multimedia services, playing games, chatting and sharing on social networks, using thousands of various softwares and so on. With the advanced development of this network infrastructure and easier access to high-speed Internet, the world wide web is becoming a truly global platform while machines and other smart objects "residing" in it are becoming increasingly capable to communicate autonomously (Miorandi et al., 2012).

Dutton (2014) emphasized that by the end of this decade already, the global network will combine the features of classic networks and networked objects. Contents and online services are already all around us, always available, speeding up communications and providing access to new applications, offering radically new ways of both work and entertainment, and indirectly - even raising the standards of living. This new level of welfare

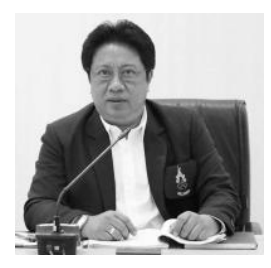

Preecha Phongpheng

$\mathrm{PhD}$, professor, Director of Office of General Education and Innovative Electronic Learning, Suan Sunandha Rajabhat University, Bangkok, Thailand

Research interests: education, management in education institutes, innovative leaning, teaching methodology Published more than 10 papers in international journals, participated in 25 International conferences, conducted researches for Ministry of Science and education of Thailand 
which integrates all the latest advances of ICTs is known as a relatively new concept of the Internet of Things, or IoT.

The very notion "Internet of Things" was introduced back in 1999 by Ashton, one of the pioneers of British technological development (Gubbi et al., 2013). The IoT aims stems from the key benefit of the Internet -- providing permanent connectivity, thus developing the ability to control remotely and share data in the physical world (Peoples et al., 2013). The Internet of Things comes from the concept Radio Frequency IDentification - RFID which is done through sensors, actuators and various gadgets such as smartphones, tablets, televisions, bracelets and smartwatches etc. Technically speaking, the Internet of Things is based on unique addressing schemes that are able to interact with each other and cooperate so that to perform the tasks assigned to them by the owner (Atzori et al., 2010).

As the IoT concept has been already discussed quite widely, the present paper aims to revise conceptually the most relevant elements of the IoT phenomenon, thus providing theoretical arguments for a new technological positioning for the Internet of Things. With this aim in mind, the article has been divided into five sections: the introduction goes first step; then three theoretical sections focus on the origin and development of the Internet of things. The fourth section offers a new technological positioning for the IoT. Final considerations and directions for further research are offered in the final part.

\section{The Origin Of The Internet Of Things}

In the course of their development in the recent couple decades, the web-based applications have undergone a range of truly radical changes. In other words, the Internet of Things did not emerge from ground zero and at once. It is vitally important to feel the differences between standard apps based on traditional Internet capabilities and a radically different set of tools that together make up the IoT.

The key aim of the IoT is to extend the physical world through 24-7 connectivity, data sharing, permanent remote control and some other, accompanying capabilities (Peoples et al., 2013). To achieve these objectives, the IoT uses the features of detection, marking/identification of things through RFID, barcodes or QR, sensors and actuators for the purposes of identification, monitoring, detection or actuation of the devices and equipment that are nearly permanently online. Such technologies allow products of physical nature store, get and send back information in a way that is radically transforming the way we do things (Dutton, 2014).

Due to the emerging growth of many technologies in the direction towards the IoT, more and more definitions of the Internet of Things are being provided in literature. The growing number of definitions actually means the growing number of difficulties with defining what this set of tools actually means. Thus, it seems to be necessary to understand the core ideas behind the IoT, its social, economic, and technical implications that may arise from its implementation and use (Zorzi et al., 2010; Vasseur et al., 2011; Dutton, 2014; Saxby, 2015). The major reason for such difficulties is because the very notion of Internet of Things combines two concepts with very different interpretations: the first part -- the Internet -- means the network able to generate so many other notions; and the second term -- things -means something very much generic, belonging to a common landscape (Atzori et al., 2010).

Differences in interpretations of the Internet of Things also come from various interests related to this phenomenon: businesser, researchers and public regulatory authorities - they 


\section{INTERNET AS A NEW TECHNOLOGICAL POSITION}

are approaching this phenomenon according to their own line of actions, their own pragmatic interests and also their own purposes, the latter can be more Internet-oriented or more thingsoriented (Brody et al., 2015). Therefore, the Internet of Things essentially means the worldwide network of interconnected smart objects which can be instantly addressed through standard communication protocols ( $\mathrm{Li}$ et al., 2014). The number of such smart objects can be truly indefinite, and their growing number means that collection, exchange, storage and interpretation of information can be carried out for multiple sources at the same time, originating from the activities of both machines and people. All of the above thus equips us all with a brand new outlook on technologies as such (Ashraf et al., 2015).

Stemming from all these, different visions on the Internet of Things, this article adopts the definition of Li et al. (2014) who defined the IoT as a set of applications enabled by the Internet but based still on physical objects and the environment integrated into the information network. The IoT consists of protocols and the related technologies that together allow various elements communicate through e-communication channels, wired and/or wireless (Valéry, 2012).

Finally, as noted by Dutton (2014) the IoT allows electronic information be transmitted through various physical objects, in a manner similar to wireless networks transmitting electronic signals and creating a truly new dimension in the design and use of the whole Internet. There is an interesting, still open question in this regard: is the IoT a really new technological generation or is it only a reorganised way of using resources already available online anyways. As we are aiming here to contribute to better understanding of this phenomenon, following, the essentially innovative elements of the phenomenon in question will be discussed below.

\section{The Internet Of Things As A New Technological Position}

The next big step in innovation of the whole society would be full-scale implementation of the Internet of Things which will be connecting not only people, but also machines and other smart objects through wireless technologies (Dutton, 2014). Under the IoT, communication between people and smart objects would be possible under any circumstances and location (Roman et al., 2011). Since the Internet of Things assumes a whole variety of various devices connected together, it becomes possible to perceive instantly all changes within the so-called smart environment (Chabridon et al., 2014). Despite the fact that the IoT clearly has a whole range of brand new technological features, there are still debates whether this is really a technological breakthrough, or only a purely technical initiative aimed at promoting and selling products and services which are only presented as innovations. And actually, there are also people who consider it to be some sort of a deviation from the already existing technological systems. Various factors shaping the technological positioning of the Internet of Things, such as wireless connection, sensors and RFID technologies are essentially not new, actually, but in case of the IoT they are being combined in brand new ways (Dutton, 2014).

Previously, all technologies used to be developed with the key objective to connect people by means of conferencing and electronic messages, however, relatively recently their development has been reshifting onto connecting devices with other devices. In such a way, the IoT shifts the focus more on technological applications. In other words, if previously technological relations used to be established as person-thing or thing-person, now they are 
increasingly more like thing-thing, thus, the whole environment of smart communications is being reconfigured (Li et al., 2014).

This changed vision has given rise to new business models and solutions, partially created with the help of the IoT or by its means. These include smart cities, smart transportation, smart houses, smart technologies in manufacturing and logistics, security, healthcare and environmental management. For this very reason, some of the experts even state that the Internet of Things is the greatest innovation in communication since the invention of the world wide web (Valéry, 2012; Li et al., 2014; Dutton, 2014).

Social and organizational impacts that the Internet of Things potentially has on the ICTs overall can actually reconfigure the way we deal with information, do business, provide services or order them, and use Internet technologies overall (Pang et al., 2015). Such transformations might seem to be too radical first but they can be actually explained as follows.

First of all, flexibility of the IoT supported by various combinations of technological solutions can actually strengthen the capacity of all technologies involved, extend their life cycle and expand the production scale so that to enter the new areas of their potential application (Dutton, 2014).

Secondly, it would be important to emphasise that the IoT does not necessarily means something entirely new. The Internet of Things aims to make things closer in real time and to make them more convenient overall.

Third, the Internet of Things places data sharing at the core of its own applicability, thus making data integration the most essential element in the functioning of different sectors and services that already exist of will be created in the near future (Dutton, 2014; Ashraf et al., 2015). At the same time, the very idea of sharing data collected for initially one purpose so that to support another purpose raises quite a lot of questions of both ethical and practical nature. Still, combining data from different individuals seems to be the key to full-scale functioning of the IoT (Dutton, 2014).

\section{The Future Of IoT}

From the economic perspective solely, the IoT is a milestone in the development of digital economy, and it may actually get out of control if not standardised in the near future already. There are already some issues being accumulated -- regarding user privacy, data protection and other social issues related to various aspects of personal identification. At the same time, technological advance caused by rapid development of the IoT can boost the development of various other industries, thus making them fully technically and financially viable.

There is also another perspective of looking at the Internet of Thing - the perspective of usefulness. In its context, the IoT can be viewed as a consolidated trend of technological improvements and radical innovations. Thus, it comes as no surprise that computer and Internet-related industries are heavily investing in this field, despite the fact that there are still much speculations as to which areas would be able to achieve stability by means of using the IoT technologies. 


\section{INTERNET AS A NEW TECHNOLOGICAL POSITION}

\section{Conclusions}

Our discussion above was aimed at reaching better understanding of what the Internet of Things represents as a relatively new technological concept emerging from the Internet and several related technologies. The central idea behind the IoT is connecting people with each other and also with a range of smart useful objects.

The absolute majority of the tools involved in making the Internet of Things happen are not new to us as such. However, the Internet of Things is connecting these smart objects (and people) on a brand new level of sophistication and data analysis. Moreover, already today it is quite ready to connect transportation systems, distribution systems, and even entire cities into a single reality. This means that the IoT provides a radically new scale of development which is based on the technological potential of the millions of the Internet-enabled devices.

At the same time, looking back on the already available literature on this subject, we could assume that new generation of research should focus more on the issues of financial viability of the Internet of Things technologies and the creation of radically new business models to provide sustainability for all businesses and services based on this technology. Also, if we want the Internet of Things to stay, we would also need research that would help building or revising public policies, laws and regulations. Moreover, subsidies from both governments and international organisations could help with regulating the use of such applications guaranteeing extra security of information, stronger privacy and freer access to such services at the same time.

In this context, as emphasized by Dutton (2014), the role and contribution of the IoT across all sectors of the economy and society needs to be valued as special. Other authors also discussing this issue including (Valéry et al., 2012, Dutton, 2014; Pang et al., 2015) same as our paper, actually, conclude that the Internet of Things should be understood as a new technological level that opens up brand new and significantly improved technical and technological possibilities. These newer possibilities can be applied in different contexts, but first of all in business sectors and in our homes.

\section{References:}

Ashraf, Q. M., \& Habaebi, M. H. (2015). Autonomic schemes for threat mitigation in Internet of Things. Journal of Network and Computer Applications, 49, 112-127.

Atzori, L., Iera, A., \& Morabito, G. (2010). The internet of things: A survey. Computer networks, 54(15), 2787-2805.

Brody, P., \& Pureswaran, V. (2015). The next digital gold rush: how the internet of things will create liquid, transparent markets. Strategy \& Leadership, 43(1), 36-41.

Chabridon, S., Laborde, R., Desprats, T., Oglaza, A., Marie, P., \& Marquez, S. M. (2014). A survey on addressing privacy together with quality of context for context management in the internet of things. Annals of telecommunications-annales des télécommunications, 69(1-2), 47-62.

Dutton, W. H. (2014). Putting things to work: social and policy challenges for the Internet of things. Info, 16(3), 1-21.

Gubbi, J., Buyya, R., Marusic, S., \& Palaniswami, M. (2013). Internet of Things (IoT): A vision, architectural elements, and future directions. Future generation computer systems, 29(7), 16451660.

Li, S., Da Xu, L., \& Zhao, S. (2015). The internet of things: a survey. Information Systems Frontiers, 17(2), 243-259. 
Miorandi, D., Sicari, S., De Pellegrini, F., \& Chlamtac, I. (2012). Internet of things: Vision, applications and research challenges. Ad Hoc Networks, 10(7), 1497-1516.

Pang, Z., Zheng, L., Tian, J., Kao-Walter, S., Dubrova, E., \& Chen, Q. (2015). Design of a terminal solution for integration of in-home health care devices and services towards the Internet-ofThings. Enterprise Information Systems, 9(1), 86-116.

Peoples, C., Parr, G., McClean, S., Scotney, B., \& Morrow, P. (2013). Performance evaluation of green data centre management supporting sustainable growth of the internet of things. Simulation Modelling Practice and Theory, 34, 221-242.

Saxby, S. (2015). The 2014 CLSR-LSPI Lisbon seminar on 'the digital citizen'-Presented at the 9th International Conference on Legal, Security and Privacy Issues in IT Law (LSPI) 15- 17 October 2014, Vieira De Almeida \& Associados, Lisbon, Portugal. Computer Law \& Security Review, 31(2), 163-180.

Valéry, N. (2012). Welcome to the thingternet: things, rather than people, are about to become the biggest users of the internet. The Economist, 21.

Vasseur, J., Agarwal, N., Hui, J., Shelby, Z., Bertrand, P., \& Chauvenet, C. (2011). RPL: The IP routing protocol designed for low power and lossy networks. Internet Protocol for Smart Objects (IPSO) Alliance, 36.

Zorzi, M., Gluhak, A., Lange, S., \& Bassi, A. (2010). From today's intranet of things to a future internet of things: a wireless-and mobility-related view. IEEE Wireless Communications, 17(6).

Paper submitted

Paper accepted for publishing

Paper published online
27 March 2019

14 May 2019

25 June 2019 